\title{
Assisting clinical decision making: tools, rules and teams
}

\section{DOI: 10.7861/clinmed.ed.21.2.1}

Clinical practice requires finessing between the scientifically ideal and the practically deliverable. A journal like ClinMed exists to help provide the reader with information to meet this balance as reflected by the message in the article by Baker et al. ${ }^{1}$ The second iteration of the National Early Warning Score (NEWS2) is a widely used clinical scoring system to enable the early detection of clinical deterioration. The variable rate of progression of acute presentations with COVID-19 raises the question of whether NEWS2 can pre-emptively identify clinical deterioration in patients hospitalised with COVID-19. A cut-off score with high sensitivity for detecting deterioration was identified, with the limitation of having a relatively high false-trigger rate.

Analogous to the use of scoring systems like NEWS2, decision tools to assist decision making in common clinical scenarios have emerged. Acute non-traumatic headache accounts for about $1 \%$ of admissions to emergency departments, and the decision about whether to undertake computed tomography of the head to identify subarachnoid haemorrhage is a common conundrum. Ramachandran and colleagues report a cohort study of two such decision-making tools, defining the sensitivity and specificity of these rules in terms of diagnostic yield. ${ }^{2}$ As with the NEWS2 manuscript, they demonstrate the importance of using such objective clinical tools in the context of the subjective patient report.

The journal also takes pride in publishing impactful studies, even if derived from a small cohort of patients. Anwar et al, from a large intensive care unit, report on a rapidly established model of a multidisciplinary group to discuss complex patients with COVID-pneumonia. ${ }^{3}$ They define the distinctive phenotypes of this patient group, and their experience of empiric use of therapies in this dynamic clinical setting, showing better outcomes than non-multidisciplinary group managed patients. The potential for such teams to also offer continuity of patient care as well as staff influence, in terms of education and wellbeing, are also considered in this excellent manuscript.

Another paper we are delighted to feature is a review of the extremely common clinical presentation of iron deficiency without anaemia. ${ }^{4}$ Despite the high prevalence, there is no consensus on management. There is particular focus on iron deficiency in pregnancy and the preoperative situation, but tolerance and speed of supplementation are particular issues. The review by Al-Naseem et al considers the causes and clinical features of iron deficiency without anaemia and proposes an algorithm of care.

The final paper to draw particular attention to is one of those included online. De Berker et al have proposed a scoring system to predict discharge destination for patients admitted with an acute stroke. ${ }^{5}$ Using data available to all stroke units in the UK, they propose a model that emphasises the importance of pre-stroke frailty, and the tool should be an invaluable adjunct to discharge planning.

Showcasing online content has been an increasing feature of these editorials in the last year, and it leads to a final item to mention in this edition. We are actively exploring the option that future editions of ClinMed will be exclusively online. Many of you will have opted out of print already, and we agree that the Royal College of Physicians (RCP) should be at the vanguard of environmental sustainability. The RCP was the first of the royal colleges to adopt a fossil fuels divestment policy, and now feels like the time to move away from the print and postage burden made on the environment. On this topic, as with the content, your feedback is always appreciated.

Anton Emmanuel Editor-in-chief

\section{References}

1 Baker KF, Hanrath AT, Schim van der Loeff I et al. National Early Warning Score 2 (NEWS2) to identify inpatient COVID-19 deterioration: a retrospective analysis. Clin Med 2021;21:84-9.

2 Foley RW, Ramachandran S, Akintimehin A et al. Subarachnoid haemorrhage rules in the decision for acute $\mathrm{CT}$ of the head: external validation in a UK cohort. Clin Med 2021;21:96-100.

3 Anwar A, Ramos-Bascon N, Crerar-Gilbert AA, Barnes N, Madden B. A specialised cardiorespiratory team approach in the intensive care management of COVID-19 patients: benefit on mortality, diagnosis and management. Clin Med 2021;21:101-6.

4 Al-Naseem A, Sallam A, Choudhury S, Thachil J. Iron deficiency without anaemia (IDWA): a diagnosis that matters. Clin Med 2021;21:107-113.

5 De Berker $\mathrm{H}$, de Berker $\mathrm{A}$, Aung $\mathrm{H}$ et al. Pre-stroke disability and stroke severity as predictors of discharge destination from an acute stroke ward. Clin Med 2021;21:e186-191.

\begin{tabular}{lll}
\multicolumn{2}{l}{ Members of the editorial board } & \\
Cono Ariti & Kate Evans & Andrew Medford \\
Paul Belcher & Laura Ferrigan & Mehool Patel \\
Tim Chevassut & Tevfik Ismail & Gerrard Phillips \\
Tahseen Chowdhury & Vikas Kapil & Sushma Saksena \\
Richard Corbett & Neelam Kumar & Philip Smith \\
Elaine Dennison & Yash Mahida & Angela Star \\
Albert Edwards & Chris Marguerie & Cameron Swift \\
Anton Emmanuel & Martin McKee & Rhys Thomas
\end{tabular}

